\title{
Recent results on atmospheric neutrino oscillation from Super-Kamiokande
}

\author{
Yoshihisa Obayashi*i \\ Kamioka Observatory, ICRR(Institute for Cosmic Ray Research), Univ. of Tokyo \\ E-mail: oobadicrr.u-tokyo.ac.jp
}

\begin{abstract}
Super-Kamiokande is a large water Cerenkov detector which consists of 50 kton pure water as a target of observation of atmospheric, solar, supernovae and accelerator neutrino and as a source of nucleon decay. Cherenkov lights emitted by charged particles from neutrino interaction at 22.5 kton of fiducial volume are viewed by 11,146 inward-facing 20-inch photomultiplier tubes(PMTs) during the first run of the detector, SK-I. The two subsequent run periods, SK-II and SK-III had 5,182 and 11,129 PMTs respectively. The outer detector surrounding the inner volume has been instrumented with 1,885 outward-facing 8-inch PMTs and used primarily as a veto.

Although Super-Kamiokande has provided enormous amount of data and physics results [1] for more than 10 years since the observation started in 1996, DAQ electronics and computers are upgraded in September 2008. There are several motivations from physics to upgrade the electronics and online system. A wider charge dynamic range enables us improvement of energy resolution of high energy atmospheric neutrino events. A trigger window with much longer time enables us to improve muon decay electron finding efficiency in atmospheric neutrino study and nucleon decay search.

After the commissioning of new DAQ system, intensive calibration works have been performed, then, SK started its physics data taking as SK-IV in October 2008.

In the paper, atmospheric neutrino oscillation analysis results from SK-I+II+III (before electronics upgrade) data on standard 2-flavor oscillation, 3-flavor oscillation with normal and inverted hierarchy, and search for CPT violation, and Early data of atmospheric neutrino from SK-IV are reported.
\end{abstract}

35th International Conference of High Energy Physics - ICHEP2010,

July 22-28, 2010

Paris France

*Speaker.

${ }^{\dagger}$ for Super-Kamiokande Collaboration 

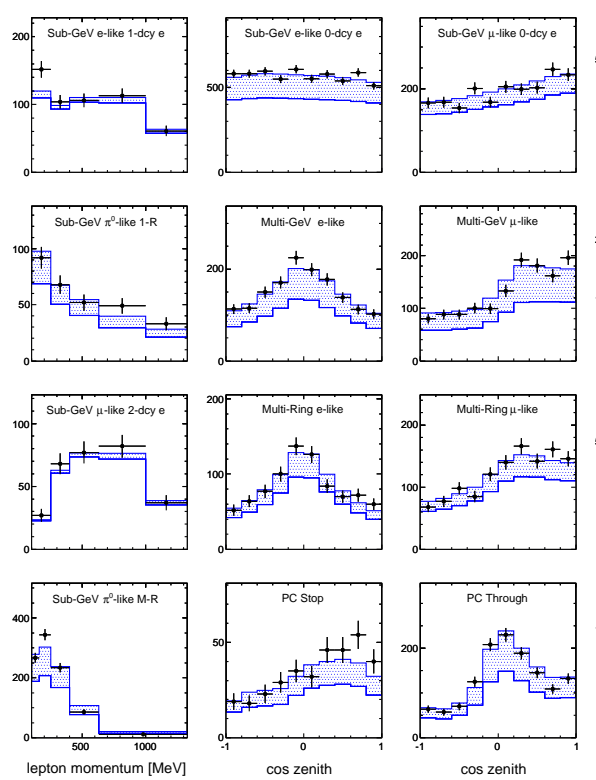

$\cos z$ zenith
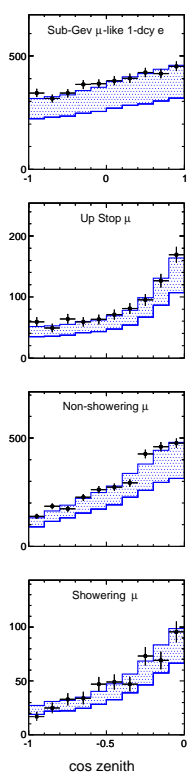

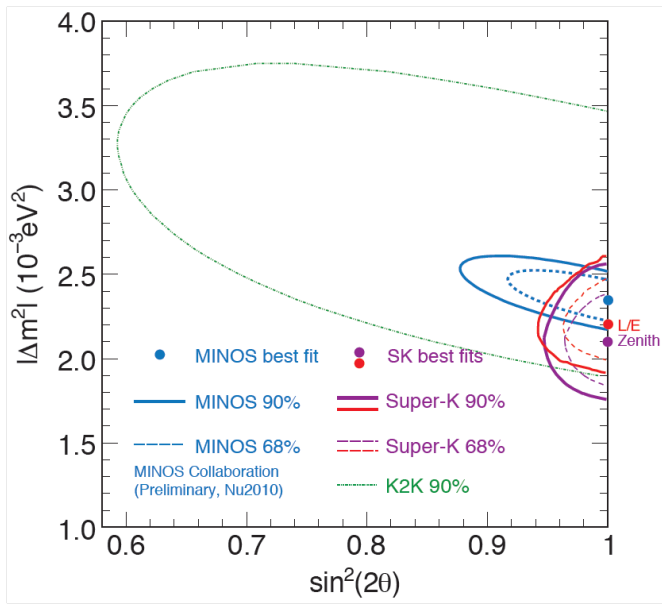

Figure 2: The allowed regions and best fit points by 2-flavor oscillation analysis. Results by Zenith angle analysis are shown by purple

Figure 1: Zenith angle and lepton momentum distributions contours and dot, results by L/E analysis are of SK-I+II+III. Black dots with statistical errors represents shown by red contours and dot. Results from MIthe data, the solid line is the oscillated MC expectation at NOS and $\mathrm{K} 2 \mathrm{~K}$ are also shown by blue and green the best fit point with the shaded region showing the an- contours, respectively.

tineutrino composition.

\section{Atmospheric neutrino data from SK-I, II and III}

The atmospheric neutrino data are divided into three categories. Fully contained (FC) events deposit all of their Cherenkov light in the inner detector (ID), Partially contained (PC) events additionally have an exiting particle that deposes energy in the outer detector (OD), and upward-going muon $(\mathrm{Up}-\mu)$ events are produced by neutrino interactions in the rock below the detector. FC events are separated into Sub-GeV $\left(\mathrm{E}_{v i s}<1.33 \mathrm{GeV}\right)$ and Multi-GeV $\left(\mathrm{E}_{v i s}>1.33 \mathrm{GeV}\right)$ then further separated based on their number of reconstructed Cherenkov rings and their particle identification. The sub-GeV single-ring e- and $\mu$ - like samples are further divided based upon their number of decay-electrons and whether or not they are $\pi^{0}$-like. Details of this selection procedure is found in [2]. The atmospheric neutrino data and MC expectation for the first three SK run period is shown in Figure1,

\section{Standard two-flavor analysis and Three flavor analysis with normal and inverted hierarchy}

A standard oscillation analysis based on the zenith angle distribution and L/E distribution is performed for SK-I+II+III atmospheric neutrino data. The details of the analysis may be found in [3], [4]. The obtained allowed regions are shown in the Figure 2. The best fit oscillation parameters obtained by the Zenith and L/E analysis are $\left(\Delta m_{23}^{2}, \sin ^{2} 2 \theta_{23}\right)=\left(2.11 \times 10^{-3}\left(\mathrm{eV}^{2}\right), 1.0\right)$ and ( $\left.2.19 \times 10^{-3}\left(\mathrm{eV}^{2}\right), 1.0\right)$, respectively. Both results of zenith angle and $\mathrm{L} / \mathrm{E}$ analysis give consistent 

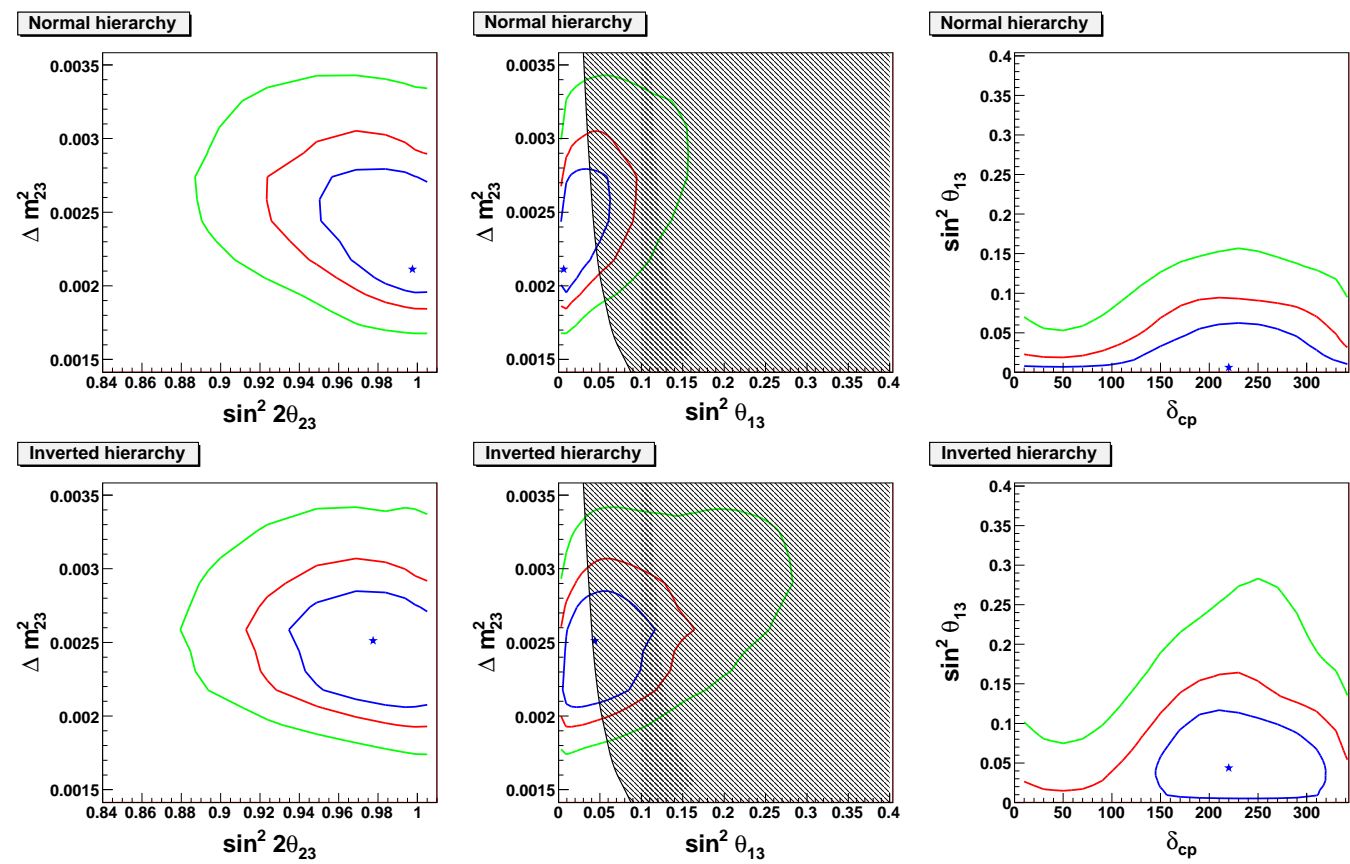

Figure 3: Allowed regions at 68\%(blue), 90\%(red), and 99\%(green) C.L. by three-flavor oscillation analysis for the SK-I+2+III data. Upper and lower figures are for the normal and invert hierarchy assumption respectively. The best fit points are shown by stars. The shaded regions in middle figures show the Chooz 90\% C.L. exclusion region.

results. Furthermore, SK provides the most stringent limit for mixing angle of $\sin ^{2} 2 \theta_{23}$ compare to recent accelerator experiments MINOS and K2K which is shown in Figure 2 as well.

A search for non-zero $\theta_{13}$ and deviations of $\sin ^{2} \theta_{23}$ from 0.5 in the three-flavor oscillation framework. Since the analysis is also sensitive for the hierarchy of neutrino mass, analysis are performed for both normal and inverted mass hierarchy. Additionally, a CP phase $\delta$ is also considered in the analysis. Detail of the analysis may be found in [2], but here all oscillation parameters are considered at a time. Obtained allowed regions and best fit points are shown in Figure 3 As a result of the analysis, consistent result to the two-flavor analysis and no significant preference on hierarchy, no significant constraint on CP phase at 90\% C.L. are obtained.

\section{A search for CPT-violation}

A search for differences in the oscillations of anti neutrinos and neutrinos in the SK-I+II+III atmospheric neutrino data has been performed. Though SK cannot distinguish $v$ from $\bar{v}$ on an event by event basis, potential difference in their oscillations would appear at a statistical level. Here, we consider ad-hoc CPT violations by testing separate two-neutrino disappearance models for $v$ and $\bar{v}$ :

$$
P\left(v_{\mu} \rightarrow v_{\mu}\right)=1-\sin ^{2} 2 \theta \sin \left(\frac{\Delta m^{2} L}{E}\right), P\left(\overline{v_{\mu}} \rightarrow \overline{v_{\mu}}\right)=1-\sin ^{2} 2 \bar{\theta} \sin \left(\frac{\Delta \bar{m}^{2} L}{E}\right) .
$$



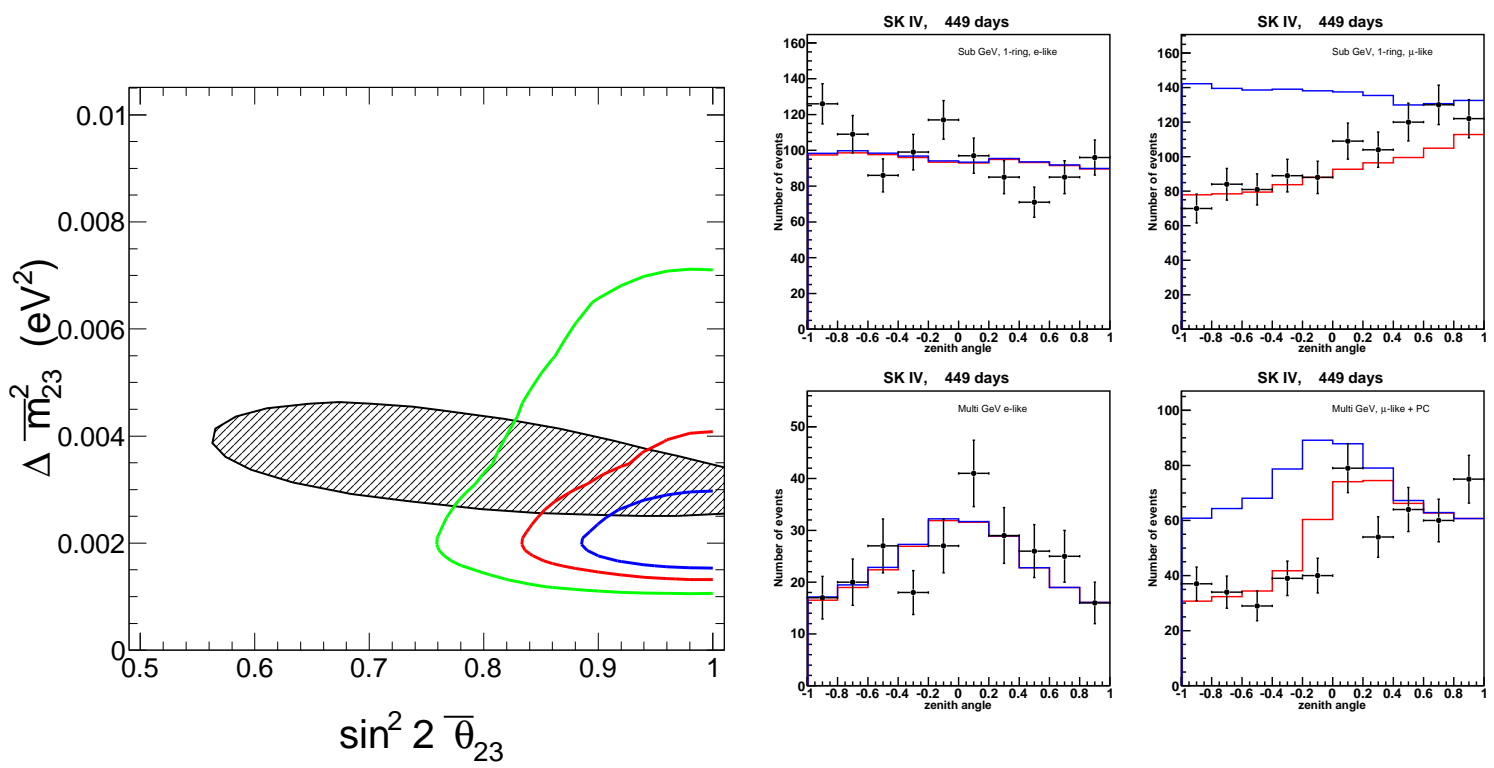

Figure 5: Zenith angle distributions for SK-IV preFigure 4: Allowed regions for the antineutrino mixing liminary data. Upper(Lower) plots are from Subparameters for the SK-I+II+III data set. The blue, red (Multi-)GeV samples, left(right) plots are from e- $(\mu$ and green contours represent the $68 \%, 90 \%$, and $99 \%$ ) like samples. Dots with statistical error bars represent allowed regions. The shaded region shows the allowed SK-IV data, blue and red lines represent expectation region from

from non-oscillation and oscillated with best fit oscillation parameters $\mathrm{MC}$, respectively.

The best fit oscillation parameters is found at $\left(\overline{\Delta m}^{2}, \sin ^{2} 2 \bar{\theta}, \Delta m^{2}, \sin 22 \theta\right)=\left(2.0 \times 10^{-3} \mathrm{eV}^{2}\right.$, $1.0,2.1 \times 10^{-3} \mathrm{eV}^{2}, 1.0$ ) with $\chi^{2}=470.6$ for 416 degrees of freedom. Allowed regions for the antineutrino oscillation parameters are shown in Figure 4 with antineutrino results from MINOS [5]. No difference in $v$ and $\bar{v}$ mixing is found in the data and the best fit in the neutrino parameters is consistent with standard two flavor results.

\section{Atmospheric neutrino data from SK-IV}

Atmospheric neutrino data is successfully accumulated after the all electronics upgrade as SKIV. Zenith angle distributions from early SK-IV atmospheric neutrino data are shown in Figure 4. Oscillation analysis results including SK-IV data will be published soon.

\section{References}

[1] Y. Fukuda et al., Phys. Rev. Lett. 81, 1562(1998)

[2] R. Wendell et al., Phys. Rev. D81, 092004 (2010).

[3] Y. Ashie et al., Phys. Rev. D71, 112005(2005).

[4] Y. Ashie et al., Phys. Rev. Lett. 93, 101801(2004).

[5] P. Vahle, in Plenary Talk at Neutrino 2010. 